\title{
Características físico-químicas e compostos aromáticos do suco de melão clarificado por microfiltração tangencial
}

\author{
Physical and chemical characteristics and aromatic compounds pulp and clarified melon juice \\ Raimundo Marcelino Silva Neto*†, Fernando Antônio Pinto de Abreu ${ }^{\dagger}$, Luiz Fernando Pellegrini \\ Pessoa ${ }^{\ddagger}$, Eduardo Mach Queiroz
}

\begin{abstract}
Resumo
Polpa e suco de melão clarificado por microfiltração tangencial foram avaliados com relação as suas características físicoquímicas e compostos aromáticos. A polpa do melão (Cucumis melo var inodorus) foi extraída, tratada com as enzimas Pectinex Ultra SP-L e Celuclast e clarificada por microfiltração à pressão média de 3,0 bar. O suco clarificado apresentouse incolor com uma leve tonalidade esverdeada. As características físico-químicas da polpa e do suco clarificado, como $\mathrm{pH}$, brix, acidez titulável, turbidez, glicose, frutose, sacarose, foram identificadas, assim como seu perfil aromático por meio de micro-extração em fase sólida (SPME) e cromatografia gasosa acoplada com espectrometria de massa (CG-EM). A microfiltração permitiu obter uma bebida de valor agregado partindo de uma matéria-prima de descarte do mercado externo, preservando suas características físico-químicas iniciais, indicando seu potencial de uso em blends de sucos de frutas tropicais. Adicionalmente, a fração retida poderá ser incorporada na polpa do melão e direcionada ao mercado de bebidas funcionais.
\end{abstract}

RM, Abreu FAP, Pessoa LFP, Queiroz EM. Características físico-químicas e compostos aromáticos do suco de melão clarificado por microfiltração tangencial. Revista Eletrônica Teccen. 2016 Jan./Jun.; 09 (1): 75-80.

Palavras-Chave: Melão; Cucumis melo; Suco clarificado; Microfiltração; Permeado.

\begin{abstract}
Pulp and melon juice clarified by crossflow microfiltration were evaluated with respect to their physical and chemical characteristics and aromatic compounds. The pulp melon (Cucumis melo var inodorus) was extracted, treated with enzymes Celuclast and Pectinex Ultra SP-L and clarified by microfiltration the average pressure of 3.0 bar. The juice clarified presented was colorless with a slight greenish tint. The physical chemical characteristics of the pulp and clarified juice, such as $\mathrm{pH}$, Brix, titratable acidity, turbidity, glucose, fructose, sucrose, were identified, as well as its flavor profile by micro solid phase extraction (SPEM) and chromatography coupled with gas mass spectrometry (GC-MS). Microfiltration yielded a beverage of value starting from raw materials from disposal of products to the foreign market, preserving their initial physical chemical characteristics, indicating its potential use in blends of tropical fruit juices. Moreover, the retained fraction may be incorporated into melon pulp, and the resulting product could be used by the functional beverage market.

Keywords: Melon; Cucumis melo; Clarified juice; Microfiltration; Permeate.
\end{abstract}

\section{Introdução}

O agronegócio do melão no Brasil vem crescendo significativamente desde o final de 1980. O Rio Grande do Norte e o Ceará se destacaram nesse cenário, produzindo em 2013 respectivamente 45\% e $38 \%$ da produção nacional, seguido pela Bahia, Rio Grande do Sul, Pernanbuco e Piauí, Cidades (2014). O melão do tipo Amarelo ainda é o preferido no Brasil, totalizando mais de $70 \%$ da área plantada. Esse tipo de melão pertence à variedade botânica Inodorus Naud., tendo como principais características a ausência de aroma, casca espessa e firme, conferindo aos frutos considerável resistência à pressão e à perda de água, aumentando sua conservação pós-colheita (Mota, Menezes, Nunes \& Rocha, 2002; Tomaz, Aroucha, Nunes, Neto \& Queiroz, 2009).
O fruto é constituído de $90 \%$ de água, sendo rico em vitaminas $\mathrm{A}, \mathrm{B}, \mathrm{B} 2$, B5 e $\mathrm{C}$, com um valor energético de 20 a $62 \mathrm{kcal} / 100 \mathrm{~g}$ de polpa. Contêm minerais como potássio, sódio e fósforo, propriedades antioxidantes além de pequenas quantidades de ácidos cítricos e málico. Seu principal açúcar é a sacarose, mas também contém frutose, glicose e rafinose em menores quantidades. Seu sabor deve-se a uma combinação de açúcares e de inúmeros compostos aromáticos, como álcoois, ésteres e cetonas (Paduan, Campos, \& Clemente, 2007; de Almeida Melo, Maciel, de Lima \& do Nascimento, 2008).

O uso de sucos clarificados como uma base neutra para a formulação de blends com frutas exóticas vem ganhando força no mercado internacional de bebidas prontas para o consumo. A tecnologia utilizada para a elaboração das chamadas bases neutras a partir de

\footnotetext{
Afiliação dos autores: † Embrapa Agroindústria Tropical

\$ Universidade Federal do Rio De Janeiro

* Endereço para correspondência: Rua Dra. Sara Mesquita, nº 2.270, Bairro Planalto do Pici, Fortaleza-CE, CEP 60511-110.

E-mail: raimundo.silva-neto@embrapa.br
} 
sucos de grande capacidade de produção agrícola surgiu da necessidade do mercado de bebidas de frutas por produtos mais saudáveis. $\mathrm{O}$ suco de melão apresentase atualmente como um alternativo potencial para $o$ suprimento do suco base direcionado à formulação de bebidas de frutas, suprindo um mercado em plena expansão. Este mercado exige características que o suco de melão naturalmente não apresenta, tais como, a limpidez, o aroma não acentuado da fruta e a estabilidade pós-processamento. Para atender essas características de produto é fundamental estabelecer um processo que associe as operações unitárias de extração da polpa, clarificação por meio do pré-tratamento enzimático acoplado à microfiltração tangencial da polpa do melão, para a obtenção do suco base de melão natural e rico em açúcares vitaminas e minerais.

Como objetivo esse trabalho visa identificar as características físico-químicas e compostos aromáticos da polpa e do suco de melão clarificado por meio da microfiltração tangencial por membranas.

\section{Material e métodos}

\section{Matéria-prima}

Melão da variedade comercial amarelo foi utilizado neste estudo por ser o mais produzido no Brasil. Além de ser inodoro, apresenta níveis de $20 \%$ de descarte por não atenderam à qualidade de exportação, devido muitas vezes, às desuniformidades físicas do fruto, ou seja, frutos maiores ou menores daqueles exigidos pelos importadores.

Foi adquirido um lote de $300 \mathrm{~kg}$ de amostra da safra de 2012/2013, oriundos de uma produção comercial localizada no Agropolo Assú-Mossoró (RN). A matériaprima foi adquirida no Centro de Abastecimento do Ceará S.A, em Fortaleza, no estágio de maturação comercial, transportada à temperatura ambiente em contentores de polietileno de capacidade de $20 \mathrm{~kg}$ e armazenadas à temperatura de $5^{\circ} \mathrm{C}$.

\section{Procedimento experimental}

Os experimentos foram realizados no Laboratório de Processos Agroindustriais da Embrapa Agroindústria Tropical, em Fortaleza-CE e na Plataforma de Tecnologia Agroalimentar da Unidade Mista de Pesquisa - UMR Qualisud, pertencente ao Centro de Cooperação Internacional de Pesquisa Agronômica para o Desenvolvimento (Cirad), localizado em Montpellier - França.

O fluxograma da Figura 1 apresenta as operações unitárias no processamento do suco de melão clarificado.

Os frutos foram pesados, lavados, sanitizados,

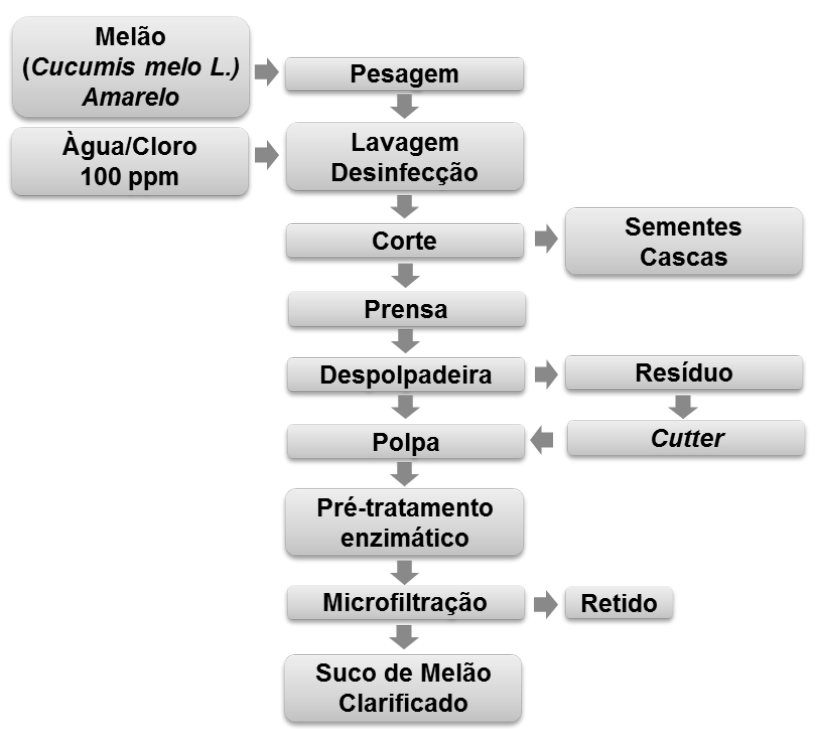

Figura 1. Fluxograma do processamento do suco de melão clarificado.

cortados em metades, retiradas as sementes, cortados em fatias, e em seguida descascadas. O mesocarpo do melão obtido das fatias descascadas foi cortado em pedaços menores, que foram levados à uma prensa tipo expeller marca INCOMAP, modelo 300, com a capacidade média de processar $300 \mathrm{~kg} / \mathrm{h}$ de frutos in natura. $\mathrm{O}$ material prensado foi despolpado em despolpadeira marca BONINA, modelo 0,25 df com peneira de 0,8 $\mathrm{mm}$, capacidade para $300 \mathrm{~kg} / \mathrm{h}$. O resíduo separado da polpa na despolpadeira foi processado em triturador marca ROBOT COUP, modelo R502 VV obtendo-se uma pasta homogênea que foi incorporada a polpa do melão obtida anteriormente na despolpadeira.

Em seguida o material foi levado para um reator e tratado enzimaticamente com 200 ppm de Pectinex Ultra SP-L e Celuclast, respectivamente, a $30^{\circ} \mathrm{C}$ durante 60 min. O reator compreende um tanque em aço inoxidável de parede dupla com capacidade de $15 \mathrm{~L}$, válvula de descarga, agitador com rotação regulada e um banhomaria que faz circular água com temperatura controlada no tanque de inox encamisado.

O material tratado enzimaticamente foi clarificado por microfiltração tangencial utilizando um piloto de laboratório TIA(Técnicas Industriais Aplicadas, Bolléne, França), com membranas tubulares tipo monocaudal, em alumina, de marca Membralox fornecida pela (Pall Exekia, Bazet, França), de 0,2 $\mu \mathrm{m}$ de diâmetro médio de poros. As pressões foram ajustadas em 5,5 e 0,5 bar de entrada e saída respectivamente, o que corresponde a uma pressão transmembranar média de 3 bar do piloto de microfiltração e a uma velocidade tangencial de 6 $\mathrm{m} / \mathrm{s}$.

A microfiltração foi realizada sem concentração do material com o sistema operando com o retorno do permeado e do retido para o tanque de alimentação, permitindo manter durante todo o experimento, uma alimentação com concentração de sólidos suspensos 
praticamente constante. As amostras de polpa e de suco de melão clarificado foram envasadas em embalagens de vidros e congelados a temperatura de $-20{ }^{\circ} \mathrm{C}$.

\section{Métodos Analíticos}

Amostras do suco de melão clarificado, do suco clarificado e concentrado e do condensado obtidas durante a evaporação foram analisadas quanto aos principais parâmetros físicos e químicos (International, 2005; AOAC, 1997): pH (utilizando pH metro ORION modelo $601 \mathrm{~A}$, com correção de temperatura), acidez total titulável (método titulométrico que utiliza uma solução de hidróxido de sódio para neutralização dos ácidos e uma solução alcoólica de fenolftaleína como indicador, sendo os resultados foram expressos em gramas de ácido málico por cem gramas de amostra), sólidos solúveis totais (leitura direta em refratômetro digital marca ATAGO modelo PR 101, com escala em graus Brix, com correção de temperatura e uma leitura máxima de $90{ }^{\circ} \mathrm{Brix}$ ), matéria seca (estufagem a $50{ }^{\circ} \mathrm{C}$ durante 24 horas, em seguida dessecação a 60 ${ }^{\circ} \mathrm{C}$ à vácuo à pressão de 0.2 bar durante 24 horas), cor (espectrofotometria de absorção atômica, utilizando o espectrofotômetro marca SPECORD modelo S 600, utilizando software WINSPECTRO, no comprimento de onda de $420 \mathrm{~nm}$ ), açúcares redutores e açúcares totais (sacarose, glicose, frutose foram monitorados por Shimadzu HPLC equipado com bombas de modelo 20AB-LC e um mostrador automático SIL-20A, acoplado com um detector de PDA década 2 Antec Leyden, Holanda), análises de compostos de aroma
SPME (cromatógrafo de tipo Agilent $6890 \mathrm{~N}$ em modo de injeção automática sobre uma coluna polar capilar DBWAX J\&W 122-7032 de $30 \mathrm{~m}$ de comprimento, 0,25 $\mathrm{mm}$ de diâmetro interno e de $0,25 \mu \mathrm{m}$ de espessura de filme, os compostos são identificados comparando-os com os espectros dos compostos conhecidos e existentes nas bases de dados NIST, Pherobase e Flavornet, a comparação é realizada por meio do cálculo do índice de retenção linear ou índice de Kovats IK).

\section{Resultados e Discussão}

A Tabela 1 apresenta as características físicoquímicas do suco de melão clarificado e do suco do melão clarificado e concentrado em diferentes condições operacionais.

Os teores de glicose, frutose e sacarose em $(\mathrm{g} / 100 \mathrm{~g})$ apresentaram valores similares com exceção àqueles obtidos na amostra do suco clarificado e concentrado a $0,2 \mathrm{bar} / 61^{\circ} \mathrm{C}$. Todas as amostras apresentaram percentuais próximos com relação à quantidade total de açúcares. A pequena diferença evidenciada nos resultados pode-se sugerir a possibilidade do processo de inversão da sacarose em contato com o calor durante a concentração do suco de melão clarificado.

Vaillant et al., (2005) trabalhando com o suco de melão cantaloupe clarificado por microfiltração e concentrado por evaporação osmótica a $55 \%$ de SST alcançaram resultados semelhantes. Onsekizoglu, Bahceci, \& Acar, (2010) concentrando suco clarificado de maçã por evaporação térmica e por membranas não

Tabela 1. Principais características físico-químicas do suco de melão clarificado e do suco do melão clarificado e concentrado em diferentes condições operacionais (em parênteses o desvio padrâo das triplicatas).

\begin{tabular}{|c|c|c|c|c|}
\hline Características & $\begin{array}{c}\text { Suco } \\
\text { clarificado } \\
(7,5 \% \text { SST })\end{array}$ & $\begin{array}{l}\text { Suco clarificado } \\
\text { concentrado } \\
\left(0,2 \text { bar } / 61^{\circ} \mathrm{C}\right) \\
(68 \% \mathrm{SST})\end{array}$ & $\begin{array}{l}\text { Suco clarificado } \\
\text { concentrado } \\
\left(0,5 \text { bar } / 77^{\circ} \mathrm{C}\right) \\
(67 \% \text { SST })\end{array}$ & $\begin{array}{c}\text { Suco clarificado } \\
\text { concentrado } \\
\left(1 \mathrm{bar} / 100^{\circ} \mathrm{C}\right) \\
(66 \% \mathrm{SST})\end{array}$ \\
\hline $\mathrm{pH}\left(20^{\circ} \mathrm{C}\right)$ & $5,6(0,1)$ & $5,6^{\#}(0,1)$ & $5,6^{\#}(0,1)$ & $5,4^{\#}(0,1)$ \\
\hline Acidez total titulável ATT(\%) & $0,05(0,0)$ & $0,05^{\#}(0,0)$ & $0,05^{\#}(0,0)$ & $0,05^{\#}(0,0)$ \\
\hline Glicose $(g / 100 g)$ & $4,28(0,0)$ & $6,35^{\#}(0,0)$ & $4,16^{\#}(0,0)$ & $4,19^{\#}(0,0)$ \\
\hline$(\%)^{\# \#}$ & $37(0,3)$ & $35(0,2)$ & $36(0,2)$ & $36(0,3)$ \\
\hline Frutose $(\mathrm{g} / 100 \mathrm{~g})$ & $2,82(0,0)$ & $5,15^{\#}(0,0)$ & $3,11^{\#}(0,0)$ & $3,29^{\#}(0,1)$ \\
\hline$(\%)^{\# \#}$ & $25(0,4)$ & $29(0,2)$ & $27(0,2)$ & $28(0,3)$ \\
\hline Sacarose (g/100g) & $4,37(0,1)$ & $6,54^{\#}(0,1)$ & $4,30^{\#}(0,0)$ & $4,28^{\#}(0,1)$ \\
\hline$(\%)^{\# \#}$ & $38(0,4)$ & $36(01)$ & $37(0,3)$ & $36(0,2)$ \\
\hline Matéria seca (\%) & $7,5(0,2)$ & $68(0,2)$ & $67(0,2)$ & $66(0,2)$ \\
\hline Atividade de água (Aw) & $0,99(0,01)$ & $0,80(0,01)$ & $0,80(0,01)$ & $0,80(0,01)$ \\
\hline Cor(420 nm) & $0,05(0,0)$ & $0,14^{\#}(0,0)$ & $0,09^{\#}(0,0)$ & $1,19^{\#}(0,0)$ \\
\hline
\end{tabular}

$\left({ }^{\sharp}\right)$ Após a diluição das amostras para o teor de 7,5\% SST (\%).

(\#\#) Percentual em relação ao total dos açúcares analisados. 
notaram decréscimos dos teores dos três principais açúcares.

A cor das amostras indicou que o suco clarificado e concentrado a $0,5 \mathrm{bar} / 77^{\circ} \mathrm{C}$ teve a intensidade da cor menos afetada quando comparada com a cor da amostra do suco clarificado, enquanto que a amostra do suco clarificado e concentrado a $1 \mathrm{bar} / 100^{\circ} \mathrm{C}$ apresentou a maior intensidade de absorbância e consequentemente o aumento do escurecimento decorrente da reação de Maillard. Esse resultado justifica-se posto que a referida amostra foi submetida à condição operacional mais agressiva com relação à temperatura e ao tempo de exposição $\left(100^{\circ} \mathrm{C} / 365\right.$ minutos). Aliado à condição operacional, a concentração de glicose maior que a da frutose contribuiu para alcançar esse resultado. A Figura 2 apresenta as amostras obtidas no processo de extração da polpa e das operações de microfiltração tangencial da polpa e da concentração do suco de melão clarificado.

Nascimento, Pinto, Freitas \& de Sousa, (2015) em seus estudos relata sobre os efeitos da relação tempo/ temperatura e concentração/pH na reação de Maillard em diferentes açúcares, afirmando que o aumento do escurecimento decorrente da reação de Maillard é favorecido pelo aumento da temperatura do processo, e que a intensidade do desenvolvimento da cor escura no comprimento de ondas $420 \mathrm{~nm}$ é a consequência mensurável mais fácil da ocorrência da reação.

A Tabela 2 apresenta os compostos aromáticos identificados em amostras do suco de melão clarificado e do suco de melão clarificado e concentrado às pressões de $0,2-0,5$ e 1,0 bar, bem como na amostra do condensado extraído durante a concentração a 1,0 bar. Os compostos estão apresentados conforme os seus respectivos grupos funcionais listados em ordem alfabética. Os, aldeídos/cetonas, álcoois, éteres e os hidrocarbonetos representam aproximadamente $54,5 \%$, $32 \%, 9 \%$, e $4,5 \%$ respectivamente, dos compostos encontrados nas amostras.

Os compostos aromáticos descritores do melão (Z)-3-nonenol, (E)-6-nonenol, (Z,Z)-3,6-nonadienol (álcoois) e o nonanal (aldeído), identificados no suco do melão clarificado, foram também identificados somente nas amostras do condensado obtido durante a concentração. Não foi identificado nenhum descritor do melão nas amostras do SMCC obtido a 0.2, 0.5 e 1,0 bar.

Os compostos aromáticos 1-octanol (álcool); 2-metilpropanal, 3-metilpentanal, penten-2-one, octanal, 3-metiltiopropanal, fenilacetaldeído (aldeídos/cetonas) e furano-2-carbaldeído (éteres), não foram identificados do suco de melão clarificado. Esses neocompostos podem ser produtos de degradação dos compostos presentes no suco do melão clarificado, possivelmente formados por meio das reações de escurecimento não enzimático, Maillard e caramelização, principalmente durante a concentração realizada a 1,0 bar.

Infere-se que esses compostos poderiam já estar presentes nas amostras do suco do melão clarificado em pequenas quantidades, entretanto a sua identificação só foi possível após a degradação dos compostos majoritários ocorrido pela ação do calor durante a concentração do suco do melão clarificado.

Baldwin, (2002); Hadi, Zhang, Wu, Zhou \& Tao, (2013) afirmaram que alguns compostos de aroma estão ligados a açúcares como os glicosídeos, mais especificamente nas frutas, o O- $\beta$-D-glicosídeos e O-diglicosídeos, sendo liberados após a clivagem da ligação da porção de açúcar durante a maturação, processamento e armazenamento, ou pela ação de enzimas, ácidos ou calor. Yajima, Sakakibara, Ide, Yanai \& Kazuo, (1985) pesquisando os componentes voláteis da melancia afirmaram que o 4-oxononal, embora ainda não tenha sido relatado como componente de aroma que ocorre naturalmente neste vegetal, foi identificado por eles como sendo um composto importante para o aroma da melancia bem como o precursor do 2-pentilfurano. Song \& Forney, (2008) em trabalho sobre produção e regulação dos voláteis em frutas afirmaram que no morango a enzima álcool aciltransferase (ATT), também identificada em melões, utiliza dentre outros álcoois, o 1-octanol para finalizar a síntese dos ésteres. Podemos sugerir que o 1-octanol identificado no SMCC e no C teria a mesma finalidade. Wyllie, Leach, Wang \& Shewfelt, (1994) identificaram 20 compostos de
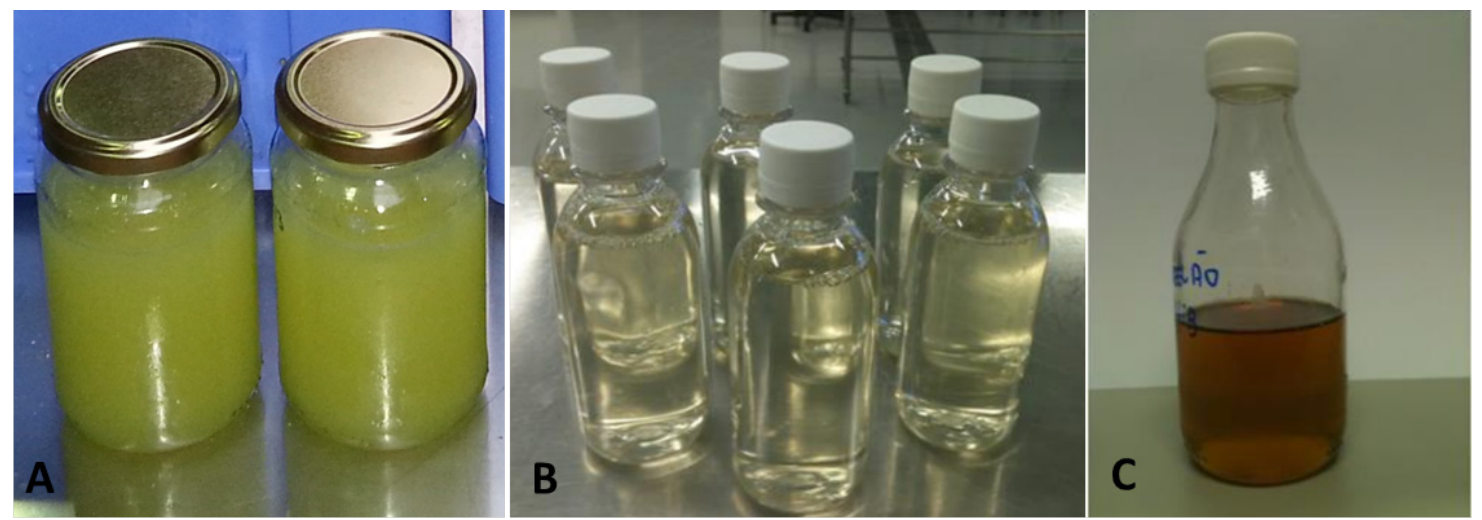

Figura 2. (A) Polpa de Melão; (B) Suco de melão clarificado e (C) Suco de melão clarificado e concentrado. 
Tabela 2. Perfil aromático dos produtos e co-produtos obtidos durante o processamento do suco de melão clarificado e concentrado.

\begin{tabular}{|c|c|c|c|c|c|c|}
\hline \multirow[t]{2}{*}{$\mathbf{N}^{0}$} & \multirow{2}{*}{$\begin{array}{c}\text { Compostos Aromáticos }^{(\mathrm{a})} \\
\text { Álcoois }\end{array}$} & \multirow[t]{2}{*}{$\mathrm{SMC}^{(\mathrm{c})}$} & \multicolumn{3}{|c|}{$\mathrm{SMCC}^{(\mathrm{d})}$} & \multirow[t]{2}{*}{$\mathbf{C}$} \\
\hline & & & 0,2 bar & 0,5 bar & 1,0bar & \\
\hline 1 & Etanol & $\mathrm{X}$ & & & & \\
\hline 2 & 1-Octen-3-ol & & & & & $\mathrm{X}$ \\
\hline 3 & 1-Octanol & & $\mathrm{X}$ & $\mathrm{X}$ & $\mathrm{X}$ & \\
\hline 4 & 2-Etilhexanol & $\mathrm{X}$ & & & & $\mathrm{X}$ \\
\hline 5 & Nonanol & $\mathrm{X}$ & & & & $\mathrm{X}$ \\
\hline 6 & (Z)-3-Nonenol (b) & $\mathrm{X}$ & & & & $\mathrm{X}$ \\
\hline 7 & (E)-6-Nonenol ${ }^{(b)}$ & $\mathrm{X}$ & & & & $\mathrm{X}$ \\
\hline \multirow[t]{2}{*}{8} & (Z,Z)-3,6-Nonadienol ${ }^{(\mathrm{b})}$ & $\mathrm{X}$ & & & & $\mathrm{X}$ \\
\hline & Aldeídos/Cetonas & & & & & \\
\hline 9 & Acetaldeido & $\mathrm{X}$ & & & & \\
\hline 10 & 2-Metilpropanal & & & & $\mathrm{X}$ & \\
\hline 11 & 3-Metilpentanal & & & $\mathrm{X}$ & & \\
\hline 12 & Etil acetato & $\mathrm{X}$ & & & & \\
\hline 13 & Pentan-2-ona & & & & & $\mathrm{X}$ \\
\hline 14 & Octanal & & & $\mathrm{X}$ & $\mathrm{X}$ & \\
\hline 15 & (E)-2-Octenal & & $\mathrm{X}$ & $\mathrm{X}$ & $\mathrm{X}$ & $\mathrm{X}$ \\
\hline 16 & 3-(Methylthio)-propanal & & & & & $\mathrm{X}$ \\
\hline 17 & 2-Metilbutanal & & & & $\mathrm{X}$ & $\mathrm{X}$ \\
\hline 18 & 3-Metilbutanal & $\mathrm{X}$ & & & $\mathrm{X}$ & $\mathrm{X}$ \\
\hline 19 & Pentanal & & & & & \\
\hline 20 & (E)-2-Butenal & & & & & $\mathrm{X}$ \\
\hline 21 & Hexanal & $\mathrm{X}$ & & & & $\mathrm{X}$ \\
\hline 22 & Nonanal $^{(\mathfrak{b})}$ & $\mathrm{X}$ & & & & $\mathrm{X}$ \\
\hline 23 & (Z)-6-Nonenal ${ }^{(\mathrm{b})}$ & $\mathrm{X}$ & & & & \\
\hline 24 & Decanal & $\mathrm{X}$ & & & & \\
\hline 25 & Fenilacetaldeído & & & & & $\mathrm{X}$ \\
\hline 26 & Benzaldeido & $x$ & & & & \\
\hline 27 & (E)-2-Nonenal ${ }^{(\mathrm{b})}$ & $x$ & & & & \\
\hline \multirow[t]{2}{*}{28} & (E,Z)-2,6-Nonadienal(b) & $x$ & & & & \\
\hline & Ésteres & & & & & \\
\hline 29 & Etil 2-metilbutanoato ${ }^{(\mathrm{b})}$ & & & & & \\
\hline \multirow[t]{2}{*}{30} & Ac Metilester octadecanoato & & & & & \\
\hline & Éteres & & & & & \\
\hline 31 & Furano-2-carbaldeido & & & & & $\mathrm{X}$ \\
\hline \multirow[t]{2}{*}{32} & 2-Pentilfurano & $x$ & & & $\mathrm{X}$ & $\mathrm{X}$ \\
\hline & Hidrocarbonetos & & & & & \\
\hline 33 & Octano & $x$ & & & & \\
\hline 34 & 1-Octeno & $x$ & & & & $\mathrm{X}$ \\
\hline 35 & (E)-2-Octeno & $x$ & & & & \\
\hline 36 & 2-Octeno & $x$ & & & & \\
\hline 37 & (Z)-2-Octeno & $x$ & & & & \\
\hline 38 & Limoneno & $x$ & & & & \\
\hline
\end{tabular}

(a) Compostos agrupados em seus respectivos grupos funcionais e estes apresentados em ordem alfabética. Foram identificados utilizando o programa MassaHunter Workstation software - Qualitative Analysis versão B.06.00 Agilent Technologies e comparados seus espectros com os existentes no NIST (National Institute of Standards and Technology).

(b) Descritores do melão pesquisados nas bases de dados acima referidas, bem como nas referências citadas. (SMC) - Suco de melão clarificado; (SMCC) - Suco de melão clarificado e concentrado a 0.2, 0.5 e 1,0 bar; (C) - Condensado obtido do SMCC a 0,5 bar. 
enxofre as amostras de Cucumis melo L. cv Makdimon e observaram que dos 7 picos identificados como odores significativos, 4 continham enxofre, os quais, S-metiltiobutanoato, 3-metiltio-propanal, 3-metiltio-acetato de propilo, e dimetil-tetrassulfureto. Cho \& Buescher, (2011) em estudos realizados sobre a degradação dos aldeídos em sucos de pepino afirmaram que o (E,Z)2,6-nonadienal e o (E)-2-nonenal, relatado na literatura como descritores do melão, são formados a partir dos ácidos linolênico e linoleico, respectivamente, quando as células são rompidas. Os mesmos autores acrescentam que esses compostos apresentam elevada instabilidade nos sucos, podendo suas perdas ser explicadas pelo aparecimento de outros aldeídos e do 2-pentilfurano.

\section{Conclusão}

O perfil dos compostos aromáticos apresenta alteração que refletiu na eliminação de alguns compostos aromáticos, entre eles os descritores do melão presente no suco clarificado e relatado na literatura, bem como na formação de neocompostos. O percentual de sacarose, frutose e glicose também evidencia leves variações devido ao processo de inversão ocorrido durante a concentração do suco clarificado, da mesma forma que a cor em consequência da reação de Maillard e da caramelização dos açúcares.

\section{Referências}

Baldwin, E. A. (2002). Fruit flavor, volatile metabolism and consumer perceptions. Fruit Quality and Its Biological Basis. CRC Press, Boca Raton, FL, 89-106.

Cho, M. J., \& Buescher, R. (2011). Degradation of cucumber flavor aldehydes in juice. Food Research International, 44(9), 2975-2977.

Cidades, (2014). Informações estatísticas 2013. Instituto Brasileiro de Geografia E Estatísticas (IBGE).

de Almeida Melo, E., Maciel, M. I. S., de Lima, V. L. A. G., \& do Nascimento, R. J. (2008). Capacidade antioxidante de frutas. Brazilian Journal of Pharmaceutical Sciences, 44(2).

El Hadi, M. A. M., Zhang, F.-J., Wu, F.-F., Zhou, C.-H., \& Tao, J. (2013). Advances in fruit aroma volatile research. Molecules, 18(7), 8200-8229.

International, AOAC. (2005). Official methods of analysis of AOAC International. AOAC International.

Mota, J. K. M., Menezes, J. B., NUNES, G. H. de S., \& Rocha, R. H. C. (2002). Qualidade e vida útil pós-colheita do melão Gold Mine produzido na época das chuvas. Revista Brasileira de Produtos Agroindustriais, 4(1), 23-28.

Nascimento, A. P. S., Pinto, A. L., Freitas, J. M. S., \& de Sousa, F. C. (2015). Efeitos da relação tempo/temperatura e concentração/pH na reação de Maillard em diferentes açúcares. Revista Brasileira de Agrotecnologia, 5(1), $1-8$.

Onsekizoglu, P., Bahceci, K. S., \& Acar, M. J. (2010). Clarification and the concentration of apple juice using membrane processes: A comparative quality assessment. Journal of Membrane Science, 352(1), 160-165.

Paduan, M. T., Campos, R. P., \& Clemente, E. (2007). Qualidade dos frutos de tipos de melão, produzidos em ambiente protegido. Revista Brasileira de Fruticultura, 29(3), 535-539.
Song, J., \& Forney, C. F. (2008). Flavour volatile production and regulation in fruit. Canadian Journal of Plant Science, 88(3), 537-550.

Tomaz, H. V. de Q., Aroucha, E. M. M., Nunes, G. H. de S., Neto, F. B., \& Queiroz, R. F. (2009). Qualidade pós-colheita de diferentes híbridos de melão-amarelo armazenados sob refrigeração. Rev. Bras. Frutic., Jaboticabal, 31(4)

Vaillant, F., Cisse, M., Chaverri, M., Perez, A., Dornier, M., Viquez, F., \& Dhuique-Mayer, C. (2005). Clarification and concentration of melon juice using membrane processes. Innovative Food Science \& Emerging Technologies, 6(2), 213-220.

Wyllie, S. G., Leach, D. N., Wang, Y., \& Shewfelt, R. L. (1994). Sulfur volatiles in Cucumis melo cv. Makdimon (muskmelon) aroma: sensory evaluation by gas chromatography-olfactometry. In ACS symposium series (USA).

Yajima, I., Sakakibara, H., Ide, J., Yanai, T., \& Kazuo, H. (1985). Volatile flavor components of watermelon (Citrullus vulgaris). Agricultural and Biological Chemistry, 49(11), 3145-3150. 